\title{
THE FREE BOUNDARY FOR ELASTIC-PLASTIC TORSION PROBLEMS ${ }^{1}$
}

BY

\author{
AVNER FRIEDMAN AND GIANNI A. POZZI
}

\begin{abstract}
Consider the variational inequality: Find $u \in K$ such that $\int_{Q} \nabla u \cdot \nabla(v$ $-u)>\mu \int_{Q}(v-u)(\mu>0)$ for any $v \in K$, where $K=\left\{w \in H_{0}^{1}(Q) ;|\nabla w|<1\right\}$ and $Q$ is a 2-dimensional simply connected domain in $R^{2}$ with piecewise $C^{3}$ boundary. The solution $u$ represents the stress function in a torsion problem of an elastic-plastic bar with cross section $Q$. The sets $E=\{x \in Q ;|\nabla u(x)|<1\}$, $P=\{x \in Q ;|\nabla u(x)|=1\}$ are the elastic and plastic sets respectively. The purpose of this paper is to study the free boundary $\partial E \cap Q$; more specifically, an estimate is derived on the number of points of local maximum of the free boundary.
\end{abstract}

0. Introduction. Consider the variational inequality described in the abstract, and denote by $V_{i}$ the vertices of $\partial Q$, i.e. the points where $\partial Q$ is not $C^{3}$. If $\partial Q$ makes a turn $>\pi$ as $V_{i}$ is crossed then $V_{i}$ is called a reentrant corner; otherwise it is a nonreentrant corner.

Let $\lambda$ be a line segment lying on $\partial Q$, say on $x_{2}=0$. Denote by $d_{x_{1}}$ the $x_{1}$-derivative of the distance function $d(x)$ to $\partial Q, x \in \bar{Q}$, and assume that the number of changes of sign from plus to minus of $d_{x_{1}}$ along $\partial Q \backslash \lambda$ is a finite number $k$. It is known [4] that the plastic set is connected to $\partial Q$ and, in fact, it is made up of "loops"

$$
\left\{\bar{x} \in \bar{Q} ; d(\bar{x}, \partial Q)=d(\bar{x}, f(s))<h(s), s \in \pi_{i}\right\}
$$

where $\pi_{i}$ are arcs along $\partial Q, \partial Q$ is given by $x=f(s)$, and $f(s)$ is the unique nearest point on $\partial Q$ to $\bar{x}$. By [3], the number of $\pi_{i}$ in $\lambda$ with mutually disjoint interior is finite. Denote by $N_{i}$ the number of points of local maximum of $h(s), s \in \pi_{i}$.

In this paper it is proved that

$$
\sum_{\pi_{i} \subset \lambda} N_{i}<k
$$

In Part I we prove this result in case all the vertices $V_{i}$ are nonreentrant corners. In Part II the general case is considered; it is also proved that a similar formula holds for the curve $r=\rho_{i}(\theta)$ representing the free boundary which is based on the reentrant corner $V_{i}($ see [4]), i.e.

$$
M_{i}<k_{i}
$$

where $M_{i}$ is the number of points of local maximum of $\rho_{i}(\theta)$ and $k_{i}$ is the number of changes of sign from plus to minus of $\partial d / \partial \theta$ along $\partial Q \backslash V_{i}$.

Received by the editos October 30, 1978.

AMS (MOS) subject classifications (1970). Primary 35R05; Secondary 35A15, 35J20, 73.35.

${ }^{1}$ This work is partially supported by National Science Foundation Grant NSF MC575-21416 A01.

() 1980 American Mathematical Society $0002-9947 / 80 / 0000-0057 / \$ 04.75$ 
The method for proving (0.1), (0.2) is based on analysis of the level curves $\partial u / \partial x_{1}=0$ and $u_{\theta}=0$, respectively, in the elastic set. This study is carried out in $\S 3$.

\section{PART I. NO REENTRANT CORNERS}

1. Preliminaries. Consider the variational inequality: find $u$ such that

$$
\int_{Q} \nabla u \cdot \nabla(v-u) d x>\mu \int_{Q}(v-u) d x \quad \forall v \in K, \quad u \in K,
$$

where $Q$ is a bounded simply connected domain in $R^{2}$ and $K$ is the convex set

$$
K=\left\{v \in W_{0}^{1,2}(Q) ;|\nabla v|<1 \text { a.e. }\right\} \text {. }
$$

We recall [2] that the solution $u$ coincides with the solution of the variational inequality (1.1) with $K$ replaced by the convex set $K_{0}$ given by

$$
K_{0}=\left\{v \in W_{0}^{1,2}(Q) ; v<d\right\}
$$

where $d=d(x)=$ distance from $x$ to $\partial Q$. Further, by [5] (also by the method of [1]) $u \in W_{\text {loc }}^{2, \infty}(\Omega)$.

For any $x^{0} \in \partial Q$, if $\partial Q$ is in $C^{3}$ in a neighborhood of $x^{0}$ then $d(x)$ is in $C^{2}$ in some $\bar{Q}$-neighborhood of $x^{0}$. By the $W^{2, p}$ theory for variational inequalities with $C^{2}$ constraint it follows that $u$ is in $W^{2, p}$ in some $\bar{Q}$-neighborhood $N$ of $x^{0}$, for any $p<\infty$. (Actually $u$ is in $W^{2, \infty}(N)$; this can be proved by extending the method of [1] (oral communication from $\mathrm{H}$. Brezis), but it will not be needed here.)

The sets

$$
E=\{x \in \bar{Q} ;|\nabla u(x)|<1\}, \quad P=\{x \in \bar{Q} ;|\nabla u(x)|=1\}
$$

are called respectively the elastic and plastic subsets of $\bar{Q}$.

We shall assume:

$\partial Q$ consists of $C^{3}$ disjoint arcs $S_{1}, \ldots, S_{\tau}$ such that the terminal endpoint $V_{i}$ of $S_{i}$ coincides with the initial

$$
\text { endpoint of } S_{i+1}\left(1<i<\tau ; S_{\tau+1} \equiv \mathrm{S}_{1}\right) \text {. }
$$

We denote by $\alpha_{i}$ the angle formed at $V_{i}$ by $S_{i}$ and $S_{i+1}$, which opens into $Q$. If $\alpha_{i}>\pi$ we call $V_{i}$ a reentrant corner and, if $\alpha_{i}>\pi$, a strict reentrant corner; if $\alpha_{i}<\pi, V_{i}$ is called a nonreentrant corner.

A point $x^{0} \in Q$ is said to belong to the ridge $R$ if, for any neighborhood $N$ of $x^{0}$, $d(x)$ is not in $W^{2, \infty}(N)$. We recall [3] that

$$
\begin{aligned}
& E \cap Q=\{x \in Q ; u(x)<d(x)\} \\
& P \cap Q=\{x \in Q ; u(x)=d(x)\}
\end{aligned}
$$

every nonreentrant corner has a $\delta$-neighborhood $G_{\delta}$

such that $G_{\delta} \cap \bar{Q}$ is elastic;

$$
R \subset E \text {. }
$$


The last fact implies that

$$
\begin{aligned}
& \text { if } x^{0} \in P \cap Q \text { then there is a unique point } y^{0} \in \partial Q \\
& \text { such that } d\left(x^{0}\right)=\left|x^{0}-y^{0}\right| ;
\end{aligned}
$$

further (see [8]; also [3])

$$
\text { the entire interval } \overline{x^{0} y^{0}} \text { is plastic. }
$$

We introduce the length parameter $s$ along $\partial Q, 0<s<L$, so that $s=0$ and $s=L$ correspond to the vertex $V_{\tau}$, and as $s$ increases the curves $S_{1}, S_{2}, \ldots$ are traced in this order. We can represent $\partial Q$ in the form $x=f(s)(0<s<L)$ where $x=\left(x_{1}, x_{2}\right), f=\left(f_{1}, f_{2}\right)$; let $s_{i}$ be the value of $s$ for which $f\left(s_{i}\right)=V_{i}, 1<i<\tau$, and write $s_{0}=0, V_{0}=V_{\tau}$.

At each point $f(s), s \neq s_{i}$, denote by $\nu(s)$ the unit inner normal to $\partial Q$ at $f(s)$, and consider the ray

$$
x=f(s)+\nu(s) \rho \quad(\rho>0) .
$$

The following facts follow from $[3, \S 2]$.

Let $s \neq s_{i}$. For any $\rho_{0}>0$, if for each $0<\rho<\rho_{0}$, the point $f(s)+\nu(s) \rho$ has a unique nearest point to $\partial Q$, then this nearest point (does not depend on $\rho$ and it) is precisely the point $f(s)$; further, the interval $l_{s}=\left\{f(s)+\nu(s) \rho, 0<\rho<\rho_{0}\right\}$ does not intersect the ridge.

Suppose next that $f(s)+\nu(s) \rho_{0}$ has more than one nearest point to $\partial Q$, and let $\rho_{1}(s)=$ inf $\rho_{0}$ for all $\rho_{0}$ with this property. Then the point $f(s)+\nu(s) \rho_{1}(s)$ is in the ridge and it is thus in $E$.

In Part I we assume that

$$
\text { all the } V_{i} \text { are nonreentrant corners. }
$$

It will be convenient to define $\nu\left(s_{i}\right)$ as a fixed unit vector at $V_{i}$ pointing into $Q$.

From (1.7), (1.9), (1.10) it then follows that

$$
P \cap Q=\left\{f(s)+\nu(s) \rho, 0<\rho<h(s), s_{i-1}<s<s_{i}, 1<i<\tau\right\}
$$

where $0<h(s)<\rho_{1}(s) ; h(s)=0$ in a neighborhood of each $s_{i}$.

By [4], if $h\left(s^{0}\right)>0$ then $h$ is in $C^{k+\beta}$ (or analytic) in a neighborhood of $s^{0}$ if $\partial Q$ is $C^{k+\beta}$ (or analytic) in a neighborhood of $f\left(s^{0}\right) ; 0<\beta<1$. These properties imply the continuity of $h(s)$ for all $s$.

It follows that there exist a countable number of disjoint intervals $\left\{\alpha_{j}<s<\beta_{j}\right\}$ such that $h(s)>0$ if and only if $\alpha_{j}<s<\beta_{j}$ for some $j$. Hence

$$
P \cap Q=\bigcup_{j}\left(P_{j} \cap Q\right)
$$

where

$$
P=\left\{f(s)+\nu(s) \rho, 0<\rho<h(s) ; \alpha_{j}<s<\beta_{j}\right\} .
$$

Each $P_{j}$ is called a plastic loop (or briefly a loop) with base $\left\{f(s), \alpha_{j}<s<\beta_{j}\right\}$; if the base belongs to some arc $\sigma, \sigma \subset \partial Q$, then we also say that $P_{j}$ is based on $\sigma$. The following fact is proved in [3] (without assuming (1.11)). 


\section{If $S_{j}$ is a line segment then there are only a finite number of loops based on $S_{j}$.}

The purpose of Part I is to obtain an estimate on the number of loops based on $S_{j}$, under the assumption (1.11).

2. Statement of the main result. We choose our notation so that $\lambda=\bar{S}_{1}$, and

$$
\lambda=\left\{\left(x_{1}, 0\right) ; 0<x_{1}<b\right\}, \quad V_{0}=(b, 0), \quad V_{1}=(0,0) ;
$$

we set $\gamma=\bar{S}_{2} \cup \cdots \cup \bar{S}_{\tau}$, so that $\gamma$ is given by $x=f(s), s_{1}<s<L$. Note that $\lambda$ is also given by $x=f(s), 0<s<s_{1}$ where $b=s_{1}, f_{1}(s)=b-s, f_{2}(s) \equiv 0$. We assume that a $Q$-neighborhood of $\lambda$ lies in $x_{2}>0$, i.e. moving along $\partial Q$ with increasing $s$, the domain $Q$ is on the right.

The distance function $d(x)$ is differentiable along $x=f(s), s \neq s_{i}$. Set

$$
\begin{gathered}
d_{1}(s)=\frac{\partial d}{\partial x_{1}}(f(s)) \quad \text { if } s \neq s_{i}, \\
d_{1}\left(s_{i}\right)=\left(\partial d / \partial x_{1}\right)\left(f\left(s_{i}+0\right)\right) \quad(1<i<\tau) .
\end{gathered}
$$

We shall assume that

$$
\begin{aligned}
& \text { the set }\left\{s ; d_{1}(s)=0,0<s<L\right\} \text { consists of a finite } \\
& \text { number of points } A_{l} \text { and intervals } J_{k} \text {. }
\end{aligned}
$$

It follows that $d_{1}(s)$ changes sign (from positive to negative or vice versa) a finite number of times. Set

$$
\begin{aligned}
& k=\text { number of times } d_{1}(s) \text { changes sign from positive to } \\
& \text { negative values as } s \text { increases from } s=s_{1} \text { to } s=L .
\end{aligned}
$$

Denote by $P_{1}, \ldots, P_{m}$ the loops based on $\lambda$. Then

$$
P_{j}=\left\{\left(x_{1}, x_{2}\right) ; 0<x_{2}<\varphi\left(x_{1}\right), a_{j}<x<b_{j}\right\}
$$

where we take $0<a_{1}<b_{1}<a_{2}<b_{2}<\ldots<a_{m}<b_{m}<b$. The function $\varphi\left(x_{1}\right)$ is positive and analytic in each interval $a_{j}<x_{1}<b_{j}$; it is continuous and equal to zero at $x_{1}=a_{j}, x_{1}=b_{j}$. We extend it by zero to the entire interval $0<x_{1}<b$.

Set

$$
\begin{gathered}
P^{\lambda}=\bigcup_{j=1}^{m} P_{j}, \quad \partial P^{\lambda}=\text { boundary of } P^{\lambda}, \\
\left.\lambda_{P}=\partial P^{\lambda} \cap \partial Q \quad \text { (the plastic part of } \lambda\right), \\
\left.\lambda_{E}=\lambda \backslash \lambda_{P} \quad \text { (the elastic part of } \lambda\right), \\
\partial_{0} P^{\lambda}=\partial P^{\lambda} \backslash \lambda_{P} \quad(\text { the free boundary of the loops based on } \lambda) .
\end{gathered}
$$

Similarly we define, for the plastic loops $P_{m+1}, P_{m+2}, \ldots$ based on $\gamma$,

$$
\begin{gathered}
P^{\gamma}=\bigcup_{j>m} P_{j}, \quad \partial P^{\gamma}=\text { boundary of } P^{\gamma}, \\
\left.\gamma_{P}=\partial P^{\gamma} \cap \partial Q \quad \text { (the plastic part of } \gamma\right), \\
\left.\gamma_{E}=\gamma \backslash \gamma_{P} \quad \text { (the elastic part of } \gamma\right), \\
\partial_{0} P^{\gamma}=\partial P^{\gamma} \backslash \gamma_{P} \quad(\text { the free boundary of the loops based on } \gamma) .
\end{gathered}
$$


Notice that $\partial_{0} P^{\lambda}=\partial P^{\lambda} \cap Q, \partial_{0} P^{\gamma}=\partial P^{\gamma} \cap Q$. Set

$$
\begin{aligned}
& \lambda_{0}=\bar{\lambda}_{E} \cup \partial_{0} P^{\lambda}, \\
& \gamma_{0}=\bar{\gamma}_{E} \cup \partial_{0} P^{\gamma} .
\end{aligned}
$$

These sets are given by $f(s)+\nu(s) h(s)$ with $0<s<s_{1}$ and $s_{1}<s<L$, respectively.

Let $\delta(s)$ be a positive valued continuous function for $0<s<L$ such that $\delta\left(s_{i}\right)=0$ for all $i$. For any $\varepsilon$ positive and sufficiently small, the curve $f(s)+$ $\nu(s)(h(s)+\varepsilon \delta(s))$ is continuous and without self-intersections, and it lies in $E \cap \bar{Q}$ (sufficiently close to the curve $f(s)+\nu(s) h(s)$ ). Since any point in $E \cap Q$ can be connected to this curve by an arc in $E \cap Q$, the set $E \cap Q$ is connected.

Since every point of $P$ can be connected to $\partial Q$ by a line segment lying in $P$, $E \cap Q$ is also simply connected.

Notice that

$$
\gamma_{0} \cup \lambda_{0}=\partial(E \cap Q)
$$

The curve $\lambda_{0}$ is also given by

$$
x_{2}=\varphi\left(x_{1}\right), \quad 0<x_{1}<b,
$$

with $\varphi\left(x_{1}\right)$ the function introduced above.

In each interval $a_{j}<x_{1}<b_{j}(1<j<m), \varphi(x)$ has a certain number $N_{j}$ of points where it achieves a local maximum (the local maximum is a local strict maximum, since $\varphi$ is analytic).

We can now state the main result of Part $I$.

THEOREM 2.1. Each $N_{j}$ is a finite number and

$$
\sum_{j=1}^{m} N_{j}<k
$$

This result immediately yields:

COROLlary 2.2. The total number of loops based on $\lambda$ is $<k$; if $m=k$ then in each interval $a_{j}<x_{1}<b_{j}$ there is a point $c_{j}$ such that $\varphi\left(x_{1}\right)$ is strictly increasing if $a_{j}<x<c_{j}, \varphi\left(x_{1}\right)$ is strictly decreasing if $c_{j}<x<b_{j}$.

Corollary 2.3. If $Q$ is a convex polygon then for every side $\lambda_{i}$ there is at most one loop based on $\lambda_{i}$ and, if the loop is given by $f(s)+\nu(s) h(s), A_{i}<s<B_{i}$, then there is a number $C_{i}, A_{i}<C_{i}<B_{i}$ such that $h(s)$ is strictly increasing if $A_{i}<s<C_{i}, h(s)$ is strictly decreasing if $C_{i}<s<B_{i}$.

This corollary is a generalization of results obtained in [3] for regular polygons.

3. Auxiliary lemmas. We assume for definiteness that $d_{1}(s)>0$ if $s>s_{1}, s-s_{1}$ small and $d_{1}(s)<0$ if $L-s$ is sufficiently small; the auxiliary lemmas will be valid without this assumption. Setting $l=2 k$, there is a partition

$$
\begin{aligned}
s_{1} & =s_{1,1}<s_{1,2}<s_{1,3}<\cdots<s_{1, m_{1}} \\
& =s_{2,1}<s_{2,2}<\cdots<s_{2, m_{2}}=s_{3,1}<s_{3,2}<\cdots<s_{l, m_{l}} \\
& =s_{l+1,1}<s_{l+1,2}<\cdots<s_{l+1, m_{l+1}}=L
\end{aligned}
$$


such that

$$
\begin{array}{rll}
d_{1}(s)>0 & \text { if } s_{1,1}<s<s_{1,2} & \left(s_{1,1}<s_{1,2}\right) \\
d_{1}(s)=0 & \text { if } s_{1,2}<s<s_{1,3} & \left(s_{1,2}<s_{1,3}\right) \\
d_{1}(s)>0 & \text { if } s_{1,3}<s<s_{1,4} & \left(s_{1,3}<s_{1,4}\right) \\
d_{1}(s)=0 & \text { if } s_{1,4}<s<s_{1,5} & \left(s_{1,4}<s_{1,5}\right) \\
d_{1}(s)>0 & \text { if } s_{1,5}<s<s_{1,6} & \left(s_{1,5}<s_{1,6}\right) \\
& \vdots & \\
d_{1}(s)<0 & \text { if } s_{2,1}<s<s_{2,2} \quad\left(s_{2,1}<s_{2,2}\right) \\
d_{1}(s)=0 & \text { if } s_{2,2}<s<s_{2,3} \quad\left(s_{2,2}<s_{2,3}\right) \\
d_{1}(s)<0 & \text { if } s_{2,3}<s<s_{2,4} \quad\left(s_{2,3}<s_{2,4}\right) \\
& : &
\end{array}
$$

Thus $d_{1}(s)$ changes sign at the points $s_{1, m_{1}}, s_{2, m_{2}}, \ldots, s_{l, m_{m}}$. The vertices $V_{i}(2<i<\tau$ - 1) need not necessarily coincide with points $f\left(s_{j, m}\right)$, i.e. $s_{i}$ may be a point in some interval $\left(s_{r, p}, s_{r, p+1}\right)$. Some of the intervals $\left[s_{i, j}, s_{i, j+1}\right]$ where $d_{1} \equiv 0$ may consist of a single point.

Consider the function

$$
\tilde{d}_{1}(s) \equiv\left(\partial d / \partial x_{1}\right)(f(s)+\nu(s) h(s)) \quad(0<s<L)
$$

at a point $s=s^{0}$ such that $s^{0} \neq s_{i}$ for $1<i<\tau$, and

$$
d_{1}\left(s^{0}\right)>0 \text {. }
$$

By continuity, $d_{1}(s)>0$ in some interval $\left|s-s^{0}\right|<\delta_{0}$. Hence some $Q$-neighborhood of $f\left(s^{0}\right)$ is given by

$$
g\left(x_{2}\right)<x_{1}<g\left(x_{2}\right)+\delta_{1}, \quad\left|x_{2}-x_{2}^{0}\right|<\delta_{1},
$$

with $x_{1}=g\left(x_{2}\right)$ being a part of $\partial Q$, and $f\left(s^{0}\right)=\left(g\left(x_{2}^{0}\right), x_{2}^{0}\right) \equiv x^{0}$.

Set $y^{0}=f\left(s^{0}\right)+\nu\left(s^{0}\right) h\left(s^{0}\right) \equiv\left(y_{1}^{0}, y_{2}^{0}\right)$. Suppose $h\left(s^{0}\right)>0$. Then $x^{0}$ is the nearest point on $\partial Q$ to $y^{0}$. It is clear that, for any $\eta$ positive and sufficiently small, the ray initiating at $y^{\eta}=\left(y_{1}^{0}-\eta, y_{2}^{0}\right)$ and in the direction $y^{0} x^{0}$ intersects $\partial Q$ at $\left(x_{1}^{\eta}, x_{2}^{\eta}\right) \equiv$ $x^{\eta}$ and $\left|x^{\eta}-y^{\eta}\right|<\left|x^{0}-y^{0}\right|$; in fact, since $g^{\prime}\left(x_{2}\right)$ is a bounded function,

$$
\left|x^{\eta}-y^{\eta}\right|<\left|x^{0}-y^{0}\right|-c \eta \quad(c>0) .
$$

This implies that $d\left(y^{\eta}\right)<d\left(y^{0}\right)-c \eta$, so that

$$
\tilde{d}_{1}\left(s^{0}\right)=d_{x_{1}}\left(y^{0}\right)>c .
$$


Since $\nabla(u-d)=0$ on the free boundary, we conclude that

$$
u_{x_{1}}\left(f\left(s^{0}\right)+\nu\left(s^{0}\right) h\left(s^{0}\right)\right)=\tilde{d}_{1}\left(s^{0}\right)>c>0 \quad \text { if } h\left(s^{0}\right)>0 .
$$

By continuity, the relation (3.5) is satisfied also at any point $s^{0}$ such that

$$
\begin{aligned}
& h\left(s^{0}\right)=0 \text {, and there exists a sequence } s^{k} \text { such that } \\
& s^{k} \rightarrow s^{0}, h\left(s^{k}\right)>0 .
\end{aligned}
$$

Suppose next that $h\left(s^{0}\right)=0$ but (3.6) is not satisfied. Then $h(s)=0$ in a neighborhood of $s^{0}$. Consequently, there exists a $Q$-neighborhood $W$ of $x^{0}$ which is elastic. Since $-\Delta u=\mu>0$ in $W, u>0$ in $W, u=0$ on $\partial Q$, the strong maximum principle gives $\left(\partial u / \partial \nu\left(s^{0}\right)\right)\left(x^{0}\right)>0$. Recalling (3.3) we deduce that $u_{x_{1}}\left(f\left(s^{0}\right)\right)>0$. We have thus proved, in general, that

$$
\text { if } d_{1}\left(s^{0}\right)>0 \text { then } \tilde{d}_{1}\left(s^{0}\right)>0, \quad u_{x_{1}}\left(f\left(s^{0}\right)+\nu\left(s^{0}\right) h\left(s^{0}\right)\right)>0 .
$$

Similarly one can show that if $d_{1}\left(s^{0}\right)<0$ then

$$
\tilde{d}_{1}\left(s^{0}\right)<0, \quad u_{x_{1}}\left(f\left(s^{0}\right)+\nu\left(s^{0}\right) h\left(s^{0}\right)\right)<0 .
$$

Finally, if $d_{1}(s)=0$ in an open interval $\alpha<s<\beta$, then $\{f(s), \alpha<s<\beta\}$ is a line segment parallel to the $x_{1}$-axis; it follows that

$$
\tilde{d}_{1}(s)=0, \quad u_{x_{1}}(f(s)+\nu(s) h(s))=0 \quad \text { if } \alpha<s<\beta .
$$

Set

$$
u_{1}(s)=\frac{\partial u}{\partial x_{1}}(f(s)+\nu(s) h(s)), \quad 0<s<L
$$

if $s \neq s_{i}$; we also set $u_{1}\left(s_{i}\right)=\varlimsup_{s \downarrow_{i}} u_{1}(s)$. Since $V_{i}$ is a nonreentrant corner, $\Delta u=$ - $\mu$ and $u<C d^{1+\alpha}(\alpha>0)$ in a $Q$-neighborhood $W_{i}$ of $V_{i}$ (by [3]). It follows that $u \in C^{1}\left(\bar{W}_{i}\right)$ and $h\left(s_{i}\right)=0$; hence $u_{1}\left(s_{i}\right)=u_{x_{1}}\left(V_{i}\right)$.

Recalling (3.1), we can sum up what we have proved so far in the following statement.

LEMMA 3.1. The assertion (3.1) holds with $d_{1}(s)$ replaced by $\tilde{d}_{1}(s)$ and by $u_{1}(s)$; further,

$$
\tilde{d}_{1}(s)=u_{1}(s)=0 \text { if } 0<s<s_{1} .
$$

The proof of Lemma 3.1 shows that the partition, into intervals of constant sign, corresponding to $\tilde{d}_{1}(s), u_{1}(s)$ is actually the same partition as for $d_{1}(s)$ (in (3.1)). This fact will not be needed later on.

Definition. The points $f(s)+\nu(s) h(s)$ for which $u_{1}(s)=0$ will be called the flat points of the solution. The set of flat points consists of a finite number of isolated points (called isolated flat points) and arcs (called flat intervals).

Notice that the flat intervals correspond to $\left[0, s_{1}\right]$ and to those intervals $\left[s_{1,2}, s_{1,3}\right]$, $\left[s_{1,4}, s_{1,5}\right], \ldots$ in (3.1) where $d_{1}(s) \equiv 0$ and which do not reduce to single points.

The function $u_{x_{1}}$ is harmonic in $E \cap Q$ and vanishes on the flat intervals of $u$. Hence it has harmonic continuation across any point $x^{0} \in Q$ of the free boundary provided $x^{0}$ lies in the interior of a flat interval. Thus, in particular, $u_{x_{1}}$ has 
harmonic continuation across $\partial_{0} P^{\lambda}$. It also has harmonic continuation across any open line segment of $\partial Q \cap \bar{E}$ which is parallel to the $x_{1}$-axis.

At each point $x^{0} \in(E \cap Q) \cup \partial_{0} P^{\lambda}$ where $u_{x_{1}}\left(x^{0}\right)=0$, we can expand the harmonic function $u_{x_{1}}$ in a (28)-neighborhood of $x^{0}$ :

$$
u_{x_{1}}=A r^{n} \cos n \varphi+\sum_{j=n+1}^{\infty}\left(A_{j} r^{j} \cos j \varphi+B_{j} r^{j} \sin j \varphi\right) \quad(r<2 \delta)
$$

where $A \neq 0, n$ a positive integer and $(r, \varphi)$ are polar coordinates about $x^{0}$. It follows that the set $\left\{x ; u_{x_{1}}(x)=0,\left|x-x^{0}\right|<\delta\right\}$ consists of $2 n$ analytic arcs

$$
r=\rho_{j}(\varphi)
$$

initiating at $x^{0}$ and terminating on $\left\{x ;\left|x-x^{0}\right|=\delta\right\}$. We shall denote by $\delta^{*}\left(x^{0}\right)$ the largest possible $\delta$ for which the expansion (3.10) is valid, and take $\delta=\delta^{*}\left(x^{0}\right)$.

Suppose $x^{0} \in E \cap Q, u_{x_{1}}\left(x^{0}\right)=0$, and consider one of the arcs (3.11). If an endpoint, say $x^{1}$, lies in $E \cap Q$, we continue it by a different arc initiating at $x^{1}$ and terminating on $\left\{x ;\left|x-x^{1}\right|=\delta^{*}\left(x^{1}\right)\right\}$. Proceeding in this way step-by-step, we obtain a piecewise analytic curve $\Gamma$ passing through $x^{0}$ and lying in $E \cap Q$; the construction stops as soon as $\Gamma$ hits $\partial(E \cap Q)$ (otherwise it continues). We parametrize $\Gamma$ by a parameter $t,-\infty<t<\infty$, and denote its equation by $x=\Gamma(t)$.

Notice that $\Gamma$ does not have self-intersections. Indeed, otherwise (since $E \cap Q$ is simply connected) there is a domain in $E \cap Q$ whose boundary is a subset of $\Gamma$. Since $u_{x_{1}}=0$ on $\Gamma, u_{x_{1}} \equiv 0$ in this domain, which is impossible.

Notice next that if $\Gamma\left(t_{n}\right) \rightarrow y^{0}$ for some sequence $t_{n} \rightarrow \infty$ (or $t_{n} \rightarrow-\infty$ ) then $y^{0} \notin E \cap Q$. Indeed, the argument of the previous paragraph shows that $y_{0} \notin \Gamma$. Now if $y^{0} \in E \cap Q$ then $\Gamma\left(t_{n}\right)$, for all large $n$, must belong to one of the analytic curves (3.11) about $y^{0}$. But then also $\Gamma(t)$, for $t>t_{n}$, lies on this arc, which we denote by $\sigma$. For any $y \in \sigma, \delta^{*}(y)>c>0$. Since in each step of the construction of $\Gamma$, we extend it from a point $y$ to a point lying on $|x-y|=\delta^{*}(y)$ (unless $\partial(E \cap Q)$ is intersected), we get, by taking $y=\Gamma\left(t_{n}\right)$ sufficiently close to $y^{0}$, that $y^{0}$ lies on $\Gamma$; a conradiction.

LEMMA 3.2. $\Gamma_{\infty} \equiv \lim _{t \rightarrow \infty} \Gamma(t)$ exists and belongs to $\partial(E \cap Q)$.

Proof. Suppose $t_{n} \rightarrow \infty, \tilde{t}_{n} \rightarrow \infty$, and

$$
\Gamma\left(t_{n}\right) \rightarrow x^{0}, \quad \Gamma\left(\tilde{t}_{n}\right) \rightarrow y^{0}, \quad x^{0} \neq y^{0} .
$$

As shown above, $x^{0}$ and $y^{0}$ must belong to $\partial(E \cap Q)$. We may assume that $t_{n}<\tilde{t}_{n}<t_{n+1}$. Introduce the curves

$$
\Gamma_{n}=\left\{\Gamma(t) ; t_{n}<t<\tilde{t}_{n}\right\}
$$

and pick a point $z^{n}$ on $\Gamma_{n}$. We may assume, for simplicity, that $z=\lim z^{n}$ exists. By the paragraph preceding Lemma 3.2 we know that $z \notin E \cap Q$. We also have

$$
z \notin\left\{\text { int } \lambda_{E}\right\} \cup \partial_{0} P^{\lambda} \text {. }
$$


Indeed, if $z \in$ int $\lambda_{0}$ then $u_{x_{1}}$ is harmonic in a neighborhood of $z$. Hence the set

$$
T=\left\{x ; u_{x_{1}}(x)=0,|x-z|<2 \delta\right\} \quad \text { (for small } \delta>0 \text { ) }
$$

consists of a finite number $l$ of analytic arcs, and therefore $T$ intersects the circle $\{|x-z|=\delta\}$ a finite number of times. Since however $z^{n} \rightarrow z, z^{n} \in \Gamma_{n}$ and (3.12) holds, $\Gamma$ enters and exits the disc $\{|x-z|<\delta\}$ an infinite number of times, a contradiction.

The same proof also shows that

$$
z \text { does not belong to the interior of any flat interval of } u \text {, }
$$

so that, in particular, $z \notin \partial_{0} P^{\lambda}$; thus (3.13) is proved. Notice next that $z$ cannot belong to any point of $\partial P^{\gamma}$ where $u_{1}\left(s^{9}\right) \neq 0$; it also cannot coincide with any point $f\left(s^{0}\right)$ of $\gamma_{E}$ for which $u_{1}\left(s^{0}\right) \neq 0$. Thus, in view of Lemma 3.1, z must belong to the finite set consisting of the isolated flat points and the endpoints of the flat intervals of $u$.

But by specifying $z^{n}$ in different positions on $\Gamma_{n}$, we may achieve any finite number of distinct limit points $z$. Since these points must belong to a given finite set of points, we get a contradiction.

Lemma 3.2 extends to $\lim \Gamma(t)$ as $t \rightarrow-\infty$. For the sake of reference we summarize:

LEMMA 3.3. Through each point $x^{0} \in E \cap Q$ where $u_{x_{1}}\left(x^{0}\right)=0$ there passes a piecewise analytic curve $\Gamma=\{\Gamma(t) ;-\infty<t<\infty\}$ contained in $E \cap Q$ along which $u_{x_{1}}=0 . \Gamma$ has no self-intersections and

$$
\Gamma_{\infty}=\lim _{t \rightarrow \infty} \Gamma(t), \quad \Gamma_{-\infty}=\lim _{t \rightarrow-\infty} \Gamma(t)
$$

exist and belong to $\partial(E \cap Q)=\gamma_{0} \cup \lambda_{0}$.

Consider a loop $P_{j}(1<j<m)$; it is given by

$$
0<x_{2}<\varphi\left(x_{1}\right), \quad a_{j}<x_{1}<b_{j} .
$$

Suppose $\beta$ is a point of local maximum of $\varphi$; then

$$
\varphi\left(x_{1}\right)<\varphi(\beta) \text { if } a_{j}<\beta-\delta_{0}<x_{1}<\beta+\delta_{0}<b_{j}
$$

for some $\delta_{0}>0$.

LeMma 3.4. There is a piecewise analytic curve $\Gamma=\{\Gamma(t), 0<t<\infty\}$ initiating at $(\beta, \varphi(\beta))$ such that $\Gamma(t) \in E \cap Q$ for all $0<t<\infty$, and $u_{x_{1}}=0$ along $\Gamma ; \Gamma$ has no self-intersections, and $\Gamma_{\infty}=\lim _{t \rightarrow \infty} \Gamma(t)$ exists and belongs to $\gamma_{0}$.

Proof. For any small $\delta>0, \delta<\delta_{0}$,

$$
\begin{aligned}
& u(\beta-\delta, \varphi(\beta))<d(\beta-\delta, \varphi(\beta))=\varphi(\beta), \\
& u(\beta+\delta, \varphi(\beta))<d(\beta-\delta, \varphi(\beta))=\varphi(\beta) .
\end{aligned}
$$

Hence $u_{x}\left(\beta-\delta_{1}, \varphi(\beta)\right)>0, u_{x}\left(\beta+\delta_{2}, \varphi(\beta)\right)<0$ for some $0<\delta_{1}, \delta_{2}<\delta$. By continuity,

$$
u_{x}\left(\beta-\delta_{1}, \varphi(\beta)+\varepsilon\right)>0, \quad u_{x}\left(\beta+\delta_{2}, \varphi(\beta)+\varepsilon\right)<0
$$

if $\varepsilon>0$ is sufficiently small. Consequently there is a number $\tilde{\delta}_{e},-\delta_{1}<\tilde{\delta_{e}}<\delta_{2}$ 
such that

$$
u_{x}\left(\beta+\tilde{\delta_{\varepsilon}}, \varphi(\beta)+\varepsilon\right)=0 \text {. }
$$

The function $u_{x_{1}}$ is harmonic in a neighborhood of $(\beta, \varphi(\beta))$ and thus has an expansion (3.10) about this point. It follows that the set $\left\{u_{x_{1}}=0\right\}$ in a neighborhood of $(\beta, \varphi(\beta))$ is given by $2 n$ analytic arcs (3.11). In view of (3.16), at least one of these arcs has a subarc initiating at $(\beta, \varphi(\beta))$, which is contained in $E \cap Q$, except for its initial point. Continuing this subarc in $E \cap Q$, the proof of the lemma (except for the last statement) now follows as in the case of the preceding lemma. The fact that $\Gamma_{\infty} \notin \lambda_{0}$ follows by noting that if $\Gamma_{\infty} \in \lambda_{0}$ then there is a domain in $E$ bounded by $\Gamma$ and a part of $\lambda_{0}$, such that $u_{x_{1}}=0$ on its boundary; hence $u_{x_{1}} \equiv 0$, which is impossible. We use here the fact that $u \in C^{1}(\bar{Q})$. (Notice that in a $\bar{Q}$-neighborhood of a vertex, $\Delta u=-\mu, u<C d^{1+\alpha}$ [3], so that $u \in$ $C^{1+\nu}, 0<\nu<\alpha$.)

4. Proof of Theorem 2.1. Suppose first that $d_{1}(s)$ on $\left(s_{1}, L\right)$ is positive near $s=s_{1}$, negative near $s=L$, and $k=1$.

Denote by $\gamma_{0}\left(s^{\prime}, s^{\prime \prime}\right)$ the portion of $\gamma_{0}$ corresponding to $s^{\prime}<s<s^{\prime \prime}$, and denote by $\gamma_{0}(s)$ the point of $\gamma_{0}$ corresponding to $s$.

Suppose there is a curve $\Gamma$ as in Lemma 3.3 such that $\Gamma_{-\infty}$ and $\Gamma_{\infty}$ both lie on $\gamma_{0}$. Thus

$$
\Gamma_{-\infty}=\gamma_{0}\left(\sigma_{1}\right), \quad \Gamma_{\infty}=\gamma_{0}\left(\sigma_{2}\right), \quad s_{1}<\sigma_{1}<\sigma_{2}<L .
$$

Denote by $\gamma_{1}$ the curve obtained from $\gamma_{0}$ by replacing $\gamma_{0}\left(\sigma_{1}, \sigma_{2}\right)$ by $\Gamma$, and parametrize $\Gamma$ by the parameter $s, \sigma_{1}<s<\sigma_{2}$.

Denote by $E_{1}$ the domain bounded by $\lambda_{0}, \gamma_{1}$ and suppose that there exists a curve of the type $\Gamma$, say $\Gamma^{1}$, in $E_{1}$, such that $\Gamma_{-\infty}^{1}, \Gamma_{\infty}^{1}$ lie in $\gamma_{1}$. We modify $\gamma_{1}$ into a curve $\gamma_{2}$ obtained by replacing the part of $\gamma_{1}$ from $\Gamma_{-\infty}^{1}$ to $\Gamma_{\infty}^{1}$ by $\Gamma^{1}$. We again parametrize $\Gamma^{1}$ by $s$ so that $\gamma_{2}$ is parametrized by $s, s_{1}<s<L$. We can repeat this process step-by-step. Note however that the points $\Gamma_{-\infty}, \Gamma_{\infty}$ cannot coincide or lie in the same flat interval of $u$ (otherwise $u_{x_{1}}=0$ in some domain). Similarly $\Gamma_{-\infty}^{1}$ and $\Gamma_{\infty}^{1}$ do not lie on an arc of $\gamma_{1}$ along which $u_{x_{1}} \equiv 0$, etc. Recalling Lemma 3.1 we conclude that the process of constructing the curves $\gamma_{j}$ must end at some $j$. Thus no curves $\Gamma^{j}$ (as in Lemma 3.3) which lie in the domain bounded by $\lambda_{0}, \gamma_{j}$ and along which $u_{x_{1}}=0$, can satisfy $\Gamma_{-\infty}^{j}, \Gamma_{\infty}^{j}$ lie on $\gamma_{j}$.

For simplicity of notation we shall assume that

$$
\gamma_{j}=\gamma_{0}
$$

thus we shall prove the theorem under the assumption that

there is no curve as in Lemma 3.3 such that both $\Gamma_{-\infty}$ and $\Gamma_{\infty}$ lie on $\gamma_{0} ;(4.2)$ the proof in case $j>0$ is the same.

Consider the curve $\Gamma$ defined in Lemma 3.4. Let $\Gamma_{\infty}=\gamma_{0}\left(s^{0}\right), s_{1}<s^{0}<L$.

Denote by $\lambda_{01}$ the part of $\lambda_{0}$ from $(0,0)$ to $(\beta, \varphi(\beta))$ and by $\lambda_{02}$ the $\operatorname{arc} \lambda_{0} \backslash \lambda_{01}$. Also, denote by $D_{1}$ the domain bounded by $\gamma_{0}\left(s_{1}, s^{0}\right), \lambda_{01}, \Gamma$ and by $D_{2}$ the domain bounded by $\gamma^{0}\left(s^{0}, L\right), \lambda_{02}, \Gamma$. 
Consider first the case where

$$
\begin{aligned}
& u_{1}(s)>0 \text { if } s_{1}<s<s^{0} \\
& u_{1}(s)<0 \text { if } s^{0}<s<L .
\end{aligned}
$$

By the maximum principle,

$$
\begin{aligned}
& u_{x_{1}}>0 \text { in } D_{1}, \\
& u_{x_{1}}<0 \text { in } D_{2} .
\end{aligned}
$$

From (4.4) it follows that $(u-d)_{x_{1}}>0$ in the subset of $D_{1}$ consisting of all points $x$ for which $d(x)=x_{2}$. This inequality easily implies that

$$
\text { the function } x_{2}=\varphi\left(x_{1}\right) \text { is monotone increasing for } 0<x_{1}<\beta \text {. }
$$

Similarly, we deduce from (4.5) that

the function $x_{2}=\varphi\left(x_{1}\right)$ is monotone decreasing for $\beta<x_{1}<b$.

It follows that there can be at most one loop and that $\varphi\left(x_{1}\right)$ has a unique strict maximum. Thus the proof of the theorem is complete in this case.

Suppose next that (4.3) is not satisfied. Assume for definiteness that for some $s^{*}$, $s^{0}<s^{*}<L$,

$$
\begin{aligned}
& u_{1}(s)>0 \text { if } s_{1}<s<s^{*}, \quad u_{1}(s) \not=0 \text { if } s^{0}<s<s^{*}, \\
& u_{1}(s)<0 \text { if } s^{*}<s<s^{*}+\delta \text { for some small } \delta>0, \\
& u_{1}(s)<0 \text { if } s^{*}<s<L .
\end{aligned}
$$

Then the assertion (4.6) follows as before.

Since $u_{1}(s)$ changes sign along $\gamma_{0}\left(s^{0}, L\right)$, there is a curve $\Gamma_{1}$ in $D_{2}$ along which $u_{x_{1}}=0$. By (4.2), $\Gamma_{1}(-\infty)$ and $\Gamma_{1}(\infty)$ cannot both lie on $\gamma_{0}\left(s^{0}, L\right)$. They also cannot both lie in $\lambda_{02}$. Hence we may take $\Gamma_{1}(-\infty) \in \lambda_{02}, \Gamma_{1}(\infty) \in \gamma_{0}\left(s^{0}, L\right)$. Let $\sigma_{1}$ be defined by $\gamma_{0}\left(\sigma_{1}\right)=\Gamma_{1}(\infty)$. If $u_{1}(s)$ changes sign along $\gamma_{0}\left(s^{0}, \sigma^{1}\right)$ (or along $\gamma_{0}\left(\sigma^{1}, L\right)$ ) then we can construct a curve $\Gamma_{2}$ along which $u_{x_{1}}=0$, lying in the region bounded by $\Gamma, \Gamma_{1}, \gamma_{0}\left(s^{0}, \sigma^{1}\right)\left(\right.$ or $\left.\gamma_{0}\left(\sigma^{1}, L\right)\right)$ and a part of $\lambda_{02}$. For this curve,

$$
\Gamma_{2}(-\infty) \in \lambda_{02}, \quad \Gamma_{2}(\infty)=\gamma_{0}\left(\sigma^{2}\right) \in \gamma\left(s^{0}, \sigma^{1}\right) \quad\left(\text { or } \gamma_{0}\left(\sigma^{2}\right) \in \gamma_{0}\left(\sigma^{1}, s^{*}\right)\right) .
$$

Again if $u_{1}(s)$ changes sign along one of the arcs $\gamma_{0}\left(s^{0}, \sigma^{2}\right), \gamma_{0}\left(\sigma^{2}, L\right)$ (or $\left.\gamma_{0}\left(\sigma^{1}, \sigma^{2}\right), \gamma_{0}\left(\sigma^{2}, L\right)\right)$ then we can construct a curve $\Gamma_{3}$, along which $u_{x_{1}}=0$, which starts on $\lambda_{02}$ and ends at the corresponding arc, at a point $\sigma^{3}$, etc. Since no two terminal points of curves $\Gamma_{i}$ can lie in the same flat interval, the process of constructing curves $\Gamma_{i}$ must terminate. This implies that there is a curve, say $\Gamma_{j}$, with $\Gamma_{j}(-\infty) \in \lambda_{02}, \Gamma_{j}(\infty)=\gamma_{0}\left(\sigma_{j}\right)$ such that $s^{0}<\sigma_{j}<s^{*}$ and $u_{1}(s) \equiv 0$ on $\gamma_{0}\left(\sigma_{j}, s^{*}\right)$. Consequently $u_{1}(s) \leqslant 0$ on $\gamma_{0}\left(\sigma_{j}, L\right)$ and $u_{1}(s)>0$ on $\gamma_{0}\left(s_{1}, \sigma_{j}\right)$.

The argument following (4.3) now shows that $\varphi\left(x_{1}\right)$ is monotone increasing for $0<x_{1}<\delta$ and monotone decreasing for $\delta<x_{1}<b$, where $\Gamma_{j}(-\infty)=(\delta, \varphi(\delta))$. This completes the proof (in case $k=1$ ) and incidentally shows (since $\delta$ must coincide with $\beta$ ) that $\Gamma_{j}$ must coincide with $\Gamma$, i.e. $s^{0}=\sigma_{j}$.

Consider now the case of general $k$. Let $\beta_{j}(1<j<N)$ be points of strict local maximum for $\varphi\left(x_{1}\right)$. Construct curves $T^{j}$ as in Lemma 3.4, which initiate at 
$\left(\beta_{j}, \varphi\left(\beta_{j}\right)\right)$; each $\Gamma^{j}$ must terminate on $\gamma_{0}$, at some point $\gamma_{0}\left(\sigma_{j}\right)$ and $\bar{\Gamma}^{i} \cap \bar{\Gamma}^{j}=\varnothing$ if $i \neq j$ (otherwise $u_{x_{1}} \equiv 0$ on the boundary of some subdomain of $E$ ). Set $\Gamma^{0}=$ $\gamma_{0}\left(s_{1}, \sigma_{1}\right), \Gamma^{N+1}=\gamma_{0}\left(\sigma_{N}, L\right)$ and denote by $D_{j}$ the domain bounded by $\Gamma^{j}, \Gamma^{j+1}, \gamma_{0}\left(\sigma_{j}, \sigma_{j+1}\right)$ and $\lambda_{0 j}$, where $\lambda_{0 j}$ is the part of $\lambda_{0}$ from $\beta_{j}$ to $\beta_{j+1}$.

In each $\gamma_{0}\left(\sigma_{j}, \sigma_{j+1}\right), 1<j \leqslant N-1$, the sign of $u_{1}(s)$ cannot be fixed (otherwise $\varphi$ would be monotone in $\left.\left(\beta_{j}, \beta_{j+1}\right)\right)$; also it cannot happen that the sign will change precisely once from plus to minus, for otherwise $\varphi\left(x_{1}\right)$ would be increasing in some interval $\left(\beta_{1}, \tilde{\beta}_{j}\right)$ and decreasing in the interval $\left(\tilde{\beta}_{j}, \beta_{j+1}\right)$ (by the proof of Theorem 2.1 in the special case of $k=1$ given above, when applied to $D_{j}$; notice that $u_{x_{1}}=0$ on $\left.\Gamma^{j}, \Gamma^{j+1}\right)$.

It follows that $u_{1}(s)$ changes sign from minus to plus in each interval $\left(\sigma_{j}, \sigma_{j+1}\right)$. Finally, $u_{1}(s)$ cannot be $<0$ in the entire interval $\left(0, \sigma_{1}\right)$ (otherwise $\varphi\left(x_{1}\right)$ would be decreasing for $\left.0<x_{1}<\beta_{1}\right)$ and similarly $u_{1}(s)$ cannot be $>0$ in the entire interval $\left(\sigma_{N+1}, L\right)$. Thus, the number of sign changes of $u_{1}(s)$ along $\gamma_{0}$ from plus to minus is at least $k$. This completes the proof.

Remark 1. If $\partial Q$ is $C^{3}$ at $V_{1}$ then a $\bar{Q}$-neighborhood of $V_{1}$ may be plastic. Theorem 2.1 extends to this case too. In this case there may be a loop based on $S_{1} \cup S_{2}$. The part of this loop which is based on $S_{1}$ is given by $0<x_{2}<\varphi\left(x_{1}\right)$, $0<x_{1}<b_{0}$. In (2.6), $N_{1}$ is the number of local maximum points of $\varphi\left(x_{1}\right)$ where $0<x_{1}<b_{0}$.

REMARK 2. If $(r, \theta)$ are the polar coordinates about the origin, then the function $u_{\theta}=x_{1} u_{x_{2}}-x_{2} u_{x_{1}}$ is harmonic in every open set where $\Delta u=-\mu$. One can then show that all the results of Part I extend to the case where $\lambda$ is an arc on a circle $x_{1}^{2}+x_{2}^{2}=R^{2}$ and the functions $d_{1}(s), \tilde{d}_{1}(s), u_{1}(s)$ are replaced by

$$
\begin{gathered}
d_{0}(s) \equiv \frac{\partial d}{\partial \theta}(f(s)), \quad \tilde{d}_{0}(s)=\frac{\partial d}{\partial \theta}(f(s)+\nu(s) h(s)), \\
\frac{\partial u}{\partial \theta}(f(s)+\nu(s) h(s)) \text { respectively. }
\end{gathered}
$$

We need here the following geometric fact:

$$
\text { if } d_{0}\left(s^{0}\right)<0 \text { then } \tilde{d}_{0}\left(s^{0}\right)<0 \text {. }
$$

To prove it, let $y^{0}=f\left(s^{0}\right), x^{0}=f\left(s^{0}\right)+\nu\left(s^{0}\right) h\left(s^{0}\right) \in Q$ so that $d\left(x^{0}\right)=\left|x^{0}-y^{0}\right|$. Denote by $\vec{\sigma}_{0}$ the direction of the tangent to $\partial Q$ at $y^{0}$ which forms an acute angle with the direction $\overrightarrow{0 y^{0}} ; 0$ the origin of the polar coordinates. Denote by $\vec{\sigma}$ the ray initiating at $x^{0}$ in the direction of $\vec{\sigma}_{0}$. Then the angle $\psi$ between $\overrightarrow{x^{0} 0}$ and $\vec{\sigma}$ satisfies $\pi / 2<\psi<\pi$. This implies that $\tilde{d}_{0}\left(s^{0}\right)<0$. Similarly, $d_{0}\left(s^{0}\right)>0$ implies $\tilde{d}_{0}\left(s^{0}\right)>0$.

\section{Part II. General Corners}

5. Loops based on a line segment. In Part II we allow some of the vertices $V_{i}$ to be reentrant corners. In this section we shall generalize Theorem 2.1 and in the next section we shall study the plastic set near a reentrant corner.

Let $V_{i}$ be a reentrant corner and denote by $\tilde{S}_{i}\left(\tilde{S}_{i+1}\right)$ the ray tangent to $S_{i}\left(S_{i+1}\right)$ at $V_{i}$ and by $l_{i}\left(l_{i+1}\right)$ the ray perpendicular to $\tilde{S}_{i}\left(\tilde{S}_{i+1}\right)$ and pointing into $Q$. Denote by $\Sigma_{i}$ the angular region with opening $<\pi$ formed by $l_{1}, l_{2}$. We introduce polar 
coordinates about $V_{i}$ so that $\tilde{S_{i}}$ is given by $\theta_{i}, \tilde{S}_{i+1}$ is given by $\theta_{i+1}, \theta_{i+1}>\theta_{i}$, $\theta_{i+1}=\alpha_{i}+\theta_{i}$. Then $\alpha_{i}>\pi$, and $\Sigma_{i}$ is given by

$$
\tilde{\theta}_{i} \equiv \theta_{i}+\frac{\pi}{2}<\theta<\theta_{i+1}-\frac{\pi}{2} \equiv \tilde{\theta}_{i+1}
$$

According to [3], if $\alpha_{i}>\pi$ (i.e. $V_{i}$ is a strict reentrant corner) then there exists a continuous positive function $\gamma(\theta), \tilde{\theta}_{i}<\theta<\tilde{\theta}_{i+1}$, such that

$$
\left\{(r, \theta) ; 0<r<\gamma(\theta), \tilde{\theta}_{i}<\theta<\tilde{\theta}_{i+1}\right\} \subset P .
$$

For each $\theta \in\left(\tilde{\theta}_{i}, \tilde{\theta}_{i+1}\right)$, set $h^{*}(\theta)=\max \{r ;(r, \theta) \in P\}$. Then, by (1.8), (1.10) it follows that the nearest point on $\partial Q$ to $\left.\left(h^{*}(\theta), \theta\right)\right)$ is $V_{i}$.

The free boundary in $Q$ consists of two parts: the first part is given by $f(s)+\nu(s) h(s)$ with $s \neq s_{i}, h(s)>0$, and the second part consists of curves

$$
r=h^{*}(\theta), \quad \tilde{\theta}_{i}<\theta<\tilde{\theta}_{i+1} \quad\left(h^{*}(\theta)>0\right)
$$

around each strict reentrant corner. The set

$$
\left\{(r, \theta) ; 0<r<h^{*}(\theta), \tilde{\theta}_{i}<\theta<\tilde{\theta}_{i+1}\right\}
$$

is called a part-loop based on $V_{i}$; it is called a loop based on $V_{i}$ if $h^{*}\left(\tilde{\theta_{i}}\right)=0$, $h^{*}\left(\tilde{\theta}_{i+1}\right)=0$. By [4], $h^{*}(\theta)$ is analytic for $\tilde{\theta}_{i}<\theta<\tilde{\theta}_{i+1}$.

The function $u$ has bounded first derivatives in $\bar{E}$, but they are not continuous at the points $V_{i}$ which are strict reentrant corners. Nevertheless, the analysis of the level curves $\Gamma$ where $u_{x_{1}}=0$ can be extended to the present case, by using the following Phragmen-Lindelof type result [6].

Let $D$ be a bounded domain in $R^{2}$ and let $x^{0}$ be a point of $\partial D$. Suppose there is a line segment $\sigma$ such that $\sigma \cap D=\varnothing$ and $x^{0}$ belongs to the closure of $\sigma$. Let $v$ be a bounded harmonic function in $D$ and let

$$
\gamma_{1}=\liminf _{x \in \partial D, x \rightarrow x^{0}} v(x, y), \quad \gamma_{2}=\limsup _{x \in \partial D, x \rightarrow x^{0}} v(x, y) .
$$

Then

$$
\gamma_{1}<\liminf _{x \in D, x \rightarrow x^{0}} v(x, y)<\limsup _{x \in D, x \rightarrow x^{0}} v(x, y)<\gamma_{2} .
$$

This result implies that $\Gamma$ cannot initiate and terminate at the same reentrant corner; similarly, $\Gamma$ cannot start on $\lambda_{0}$ and terminate at $V_{j}(j=1$ or $j=0$; see the notation of 82) even when $V_{j}$ is a reentrant corner, etc. Thus the proof of Lemmas 3.2-3.4 can be extended to the present case.

In order to extend Theorem 2.1 to the case where some of the $V_{i}$ are reentrant corners, we still have to study the sign of the function $\partial d / \partial x_{1}$ on the part-loops based at strict reentrant corners $V_{i}$.

If $S_{i} \cup S_{i+1}$ form a curve $x_{1}=l\left(x_{2}\right)$ in a neighborhood of $V_{i}$, then $\partial d / \partial x_{1}$ has a fixed sign in a $Q$-neighborhood of $V_{i}$ as well as for $x$ in the sector $\Sigma_{i}$ (provided $\left.d(x)=\left|x-V_{i}\right|\right)$. If $S_{i} \cup S_{i+1}$ is not given by $x_{1}=l\left(x_{2}\right)$ then it must have the form $x_{2}=\tilde{l}\left(x_{1}\right)$. Suppose in this case that $\tilde{S}_{i}, \tilde{S}_{i+1}$ are not tangent to the $x_{1}$-axis. Then $d_{1}(s)$ changes sign at $s=s_{i}$ (from negative to positive if $x_{1}$ is increasing with $s$ along $\partial Q$, near $V_{i}$ ). In $\Sigma_{i}$ we have the same change of sign of $\partial d / \partial x_{1}$ from negative 
to positive as one crosses the ray $l_{0}$ which initiates at $V_{i}$, is parallel to the $x_{2}$-axis and is pointing into $Q$.

If $\tilde{S}_{i}$ or $\tilde{S}_{i+1}$ is tangent to the $x_{1}$-axis (and $S_{1} \cup S_{2}$ cannot be written in the form $\left.x_{1}=l\left(x_{2}\right)\right)$ then $d_{1}(s)$ may or may not change sign as one crosses $s=s_{i}$, but the same is true of $\partial d / \partial x_{1}$ in $\Sigma_{i}$ (as one crosses $l_{0}$ ).

We conclude that the change of sign of $\partial d / \partial x_{1}$ along $\partial E$ when the corresponding nearest point of $\partial Q$ varies in a neighborhood of a strict reentrant corner $V_{i}$ is the same as the change of sign of $d_{1}(s)$ at $s=s_{1}$. Thus, the number of changes of sign of $\partial d / \partial x_{1}$ along $\partial E \backslash \lambda_{0}$, is the same as the number of changes of sign of $d_{1}(s)$ as described in \$2. This is the content of Lemma 3.1.

The proof of Theorem 2.1 can now be extended with trivial changes to yield the following.

THEOREM 5.1. Theorem 2.1 remains valid even when some of the vertices $V_{i}$ are reentrant corners.

REMARK. Theorem 5.1 extends to the case where $\lambda$ is a circular arc (cf. Remark 2 at the end of \$4).

6. Loops based on strict reentrant corners. Suppose $V_{i}$ is a strict reentrant corner. We introduce polar coordinates as in \$5. The free boundary of the part-loop based on $V_{i}$ is given by $r=h^{*}(\theta), \tilde{\theta}_{i}<\theta<\tilde{\theta}_{i+1}$. Denote by $M_{i}$ the number of points of local maximum of this function.

Let

$$
\hat{d}(s)=(\partial d / \partial \theta)(f(s)+\nu(s) h(s)) \text { along } \partial Q
$$

and let

$k_{i}=$ number of changes of the sign of $\hat{d}(s)$ from negative to positive values, as $f(s)$ moves along $S_{i+1}, S_{i+2}, \ldots, S_{\tau}, S_{1}, \ldots, S_{i}$.

THEOREM 6.1. $M_{i}<k_{i}$.

The proof is similar to the proof of Theorem 5.1 (cf. the remarks at the end of $\$ \$ 4,5)$.

In [3] the free boundary was analyzed for some special domains having axis of symmetry: regular polygons, cracked disc, and cross-shaped domain; the monotonicity of the free boundary was also derived. All these results can also be obtained by using Theorems 2.1, 5.1 and 6.1. In fact, the present theorems can be applied to much more general domains than in [3]. We consider, as an example, an $L$-shaped domain

$$
\begin{gathered}
Q=Q_{1} \cup Q_{2}, \quad Q_{1}=\left\{\left(x_{1}, x_{2}\right) ;-b<x_{1}<a, 0<x_{2}<c\right\}, \\
Q_{2}=\left\{\left(x_{1}, x_{2}\right) ; 0<x_{1}<a,-d<x_{2}<0\right\} .
\end{gathered}
$$

Using Theorem 5.1 we find that on each side there is precisely one complete loop, given by a graph with a unique point of maximum. There is also a loop based in part at $0=(0,0)$ and given by $r=h^{*}(\theta)(0<\theta<\pi / 2)$; if $h^{*}(0)>0$ then the loop extends to the region $-b<x_{2}<0$, and is given there by $0<x_{2}<\varphi\left(x_{1}\right)$ where 
$\varphi\left(x_{1}\right)$ is strictly monotone increasing for $-\alpha<x_{1}<0, \varphi(-\alpha)=0$; a similar fact holds for $\theta=\pi / 2$. Finally, $h^{*}(\theta)(0<\theta<\pi / 2)$ has at most three points of local maximum.

Consider next a $T$-shaped domain $Q$. Using Theorems 2.1, 5.1 and 6.1 we find that for each side of the boundary there exists at most one loop which is based on this side. At each reentrant corner there is a part-loop based on it and $h^{*}(\theta)$ $(0<\theta<\pi / 2)$ has at most three points of local maximum. Since the $T$ domain has an axis of symmetry, say the $x_{2}$-axis, we have $u_{x_{1}}=0$ along this axis. By applying the proof of Theorems 2.1 and 5.1 in the part of the $T$ lying in $x_{1}<0\left(x_{1}>0\right)$ we find that there cannot actually be a loop which is supported by the side of the $T$ which is parallel to the $x_{1}$-axis and which has the reentrant corner as an endpoint.

Numerical computations of the plasticity set for $T$-shaped domains are given in [7].

\section{REFERENCES}

1. H. Brezis and D. Kinderlehrer, The smoothness of solutions to nonlinear variational inequalities, Indiana Univ. Math. J. 9 (1974), 831-844.

2. H. Brezis and M. Sibony, Equivalence de deux inéquations variationnelles et applications, Arch. Rational Mech. Anal. 41 (1971), 254-265.

3. L. A. Caffarelli and A. Friedman, The free boundary for elastic-plastic torsion problems, Trans. Amer. Math. Soc. 252 (1979), 65-97.

4. L. A. Caffarelli and N. M. Riviere, The smoothness of the elastic-plastic free boundary of a twisted bar, Proc. Amer. Math. Soc. 63 (1977), 56-58.

5. On the Lipschitz character of the stress tensor when twisting an elastic-plastic bar, Arch. Rational Mech. Anal. (to appear).

6. A. Friedman and R. Jensen, Convexity of the free boundary in the Stefan problem and in the dam problem, Arch. Rational Mech. Anal. 67 (1977), 1-24.

7. R. A. Glowinski, J. L. Lions and R. Tremoliéres, Approximation numérique des solutions des inéquations en méchanique et en physique, vol. 1, Dunod, Paris, 1976.

8. T. W. Ting, Elastic-plastic torsion of simply connected cylindrical bars, Indiana Univ. Math. J. 20 (1971), 1047-1076.

Department of MATHEmatics, NorthWestern University, Evanston, Illinois 60201

Department of Mathematics, University of Pavia, Pavia, Italy 